\title{
A Note on the Radiation Conductance of an Axial Slot on a Cylinder
}

\author{
Charles M. Knop ${ }^{1}$ and Calvin T. Swift ${ }^{2}$
}

(Received October 19, 1964)

\begin{abstract}
An expression is derived for the external radiation conductance of an axial slot on a cylinder using Parseval's theorem in mode space. The result is then applied to the specific case of a thin half-wavelength axial slot and agrees identically with that obtained by J. R. Wait, using an integration of Poynting's vector over physical space in the radiation zone. A brief discussion of the two methods is then given.
\end{abstract}

Consider an axial slot of length $l$ and width $w=a \phi_{0}$ cut in an infinitely long, perfectly conducting cylinder as depicted in figure 1. From the work of Wait [1959], it follows that if the electric fields over the cylinder surface $\rho=a$ are specified as

$$
\begin{aligned}
& E_{\phi}(a, \phi, z)=\left\{\begin{array}{l}
0 \\
E_{0} \cos \left(\frac{\pi \mathrm{z}}{l}\right), \\
\text { off slot }
\end{array}\right. \\
& E_{z}(a, \phi, z)=0
\end{aligned}
$$

then the fields produced at any point $(\rho, \phi, z)$ for $\rho \geqslant a$ are given by eqs (342) through (347) of Wait [1959] with $c_{m}=0$ and with $d_{m}$ given by

$$
d_{m}=\frac{\bar{E}_{\phi}}{j \omega \mu_{0} u_{0} H_{m}^{(2) \prime}\left(u_{0} a\right)}
$$

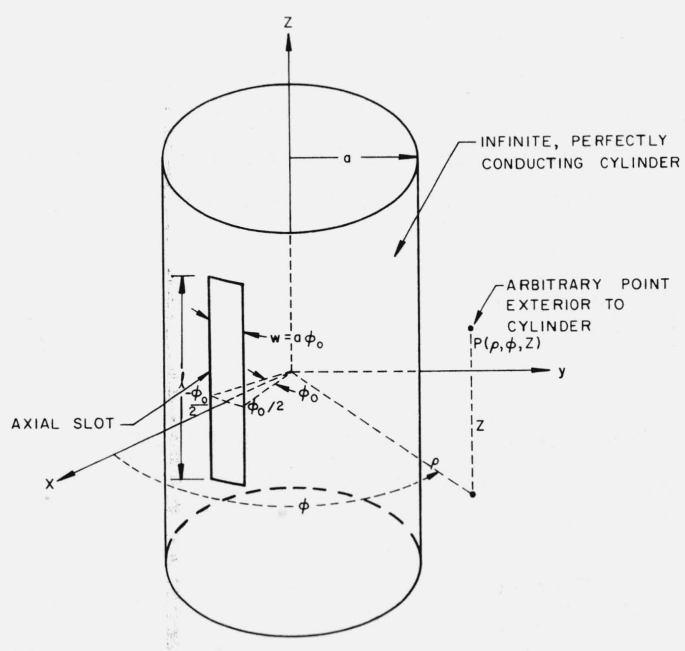

Figure 1. Axial slot on a cylinder.

1 NESCO, Pasadena, Calif.

${ }^{2}$ NASA, Langley Research Center, Langley Station, Hampton, Va. 
with all symbols defined in Wait and where $\bar{E}_{\phi}$ is the cylindrical Fourier transform of (1) defined by

$$
\bar{E}_{\phi m}(h) \equiv \bar{E}_{\phi}=\frac{1}{(2 \pi)^{2}} \int_{-\pi}^{\pi} \int_{-\infty}^{\infty} E_{\phi}(a, \phi, z) e^{j h z} e^{j m \phi} d z d \phi
$$

which becomes

$$
\bar{E}_{\phi}=\frac{-E_{0}\left(\frac{w}{a}\right)}{2 \pi} \frac{\cos \left(\frac{h l}{2}\right)}{l\left(h^{2}-\pi^{2} / l^{2}\right)} \frac{\sin \left(m \phi_{0} / 2\right)}{\left(m \phi_{0} / 2\right)} .
$$

It is noted that $E_{\phi m}(h)$ is written for brevity as $\bar{E}_{\phi}$ and is a function of $m$ and $h$. The axial magnetic field produced on the cylindrical surface $\rho=a$ by the specified electric fields (1) and (2) is then, from (345) of Wait [1959].

$$
H_{z}(a, \phi, z)=\Gamma\left(\bar{H}_{z}\right)=\sum_{m=-\infty}^{\infty} \int_{-\infty}^{\infty} \bar{H}_{z} e^{-j^{h z}} d h e^{-j^{m \phi}}
$$

where $\bar{H}_{z m}(h)$, written for brevity as $\bar{H}_{z}(h)$, is given by

$$
H_{z m}(h) \equiv \bar{H}_{z}=\frac{u_{0} H_{m}^{(2)}\left(u_{0} a\right)}{j \omega \mu_{0} H_{m}^{(2)}\left(u_{0} a\right)} \bar{E}_{\phi}
$$

Since the $z$ component of electric field is assumed to be zero over the slot, the real power which flows through the slot into the exterior is given by

$$
P=\frac{1}{2} \iint_{\text {slot }} \operatorname{Re}\left(E_{\phi}^{*} H_{z}\right) a d \phi d z
$$

where Re means real part of and * is a conjugate operator. The external radiation conductance, $G$, is defined by [Wait, 1955]

$$
G=2 P / V_{0}^{2}
$$

where $V_{0}=E_{0} w$ is the applied voltage across the center of the slot.

To evaluate $P$ by means of (8) requires that $H_{z}$ first be obtained via (6) and then the integration of (8) over the slot be performed. Thus, a double operation (i.e., (6) and (8)) is required. Rather than perform this extra labor use is made of Parseval's theorem, which states that $P$ can also be expressed as an integration of the cylindrical Fourier transforms of the pertinent fields over mode space, as

$$
P=\frac{1}{2}(2 \pi)^{2} a \operatorname{Re}\left\{\sum_{m=-\infty}^{m=\infty} \int_{-\infty}^{\infty} \bar{E}_{\phi}^{*} \bar{H}_{z} d h\right\} .
$$

This is nothing more than an extension of Parseval's theorem in rectangular Fourier transforms [Harrington, 1961] to cylindrical Fourier transforms, and is derived in appendix A.

Using (7) and the conjugate of (5) with (10) in (9) then gives

$$
G=\frac{1}{\omega \mu_{0} a\left(k_{0} l\right)^{2}} \operatorname{Re}\left\{-j \sum_{m=-\infty}^{\infty} \frac{\sin ^{2}\left(\frac{m \phi_{0}}{2}\right)}{\left(\frac{m \phi_{0}}{2}\right)^{2}} I_{m}\right\}
$$


with

$$
I_{m}=\int_{-\infty}^{\infty} \frac{\sqrt{1-y^{2}} \cos ^{2}\left(\frac{k_{0} l}{2}\right) H_{m}^{(2)}\left[C \sqrt{1-y^{2}}\right] d y}{\left[y^{2}-\pi^{2} /\left(k_{0} l\right)^{2}\right]^{2} H_{m}^{(2)}\left[C \sqrt{1-y^{2}}\right]}
$$

where $y=h l / k_{0} l$ is the dimensionless dummy variable of integration and $C=k_{0} a$ is the circumference of the cylinder in free space wavelengths. It must now be noted that (12) cannot be meaningfully integrated prior to specifying the proper roots of the quantity $\sqrt{1-y^{2}}$. These roots are chosen in accordance with the physical consideration that $e^{-j u_{0} \rho}$ (which represents the asymptotic wave behavior of the Hankel functions appearing in the integrand of (12) must represent outward going, bounded waves. Hence, $u_{0}=+\sqrt{{k_{0}}^{2}-h^{2}}$ for $|h|<k_{0}$ and $u_{0}=-j \sqrt{h^{2}-k_{0}^{2}}$ for $|h|>k_{0}$. Accordingly, the roots

$$
\sqrt{1-y^{2}}= \begin{cases}+\sqrt{1-y^{2}} & |y|<1 \\ -j \sqrt{y^{2}-1} & |y|>1\end{cases}
$$

must be chosen.

This recognition in conjunction with the identities

$$
\begin{aligned}
& J_{m}(-j x)=j^{m}(-1)^{m} I_{m}(x) \\
& Y_{m}(-j x)=j^{m}\left[-\frac{2}{\pi} K_{m}(x)-j(-1)^{m} I_{m}(x)\right]
\end{aligned}
$$

giving

$$
\begin{aligned}
& H m^{(2)}(-j x)=j \frac{2}{\pi} j^{m} K_{m}(x) \\
& H_{m}^{(2)^{\prime}}(-j x)=-\frac{2}{\pi} j^{m} K_{m}^{\prime}(x)
\end{aligned}
$$

shows that only the integration over $y$ in the interval -1 to 1 contributes to $G$. Use of the Wronskian relation $J_{m}(x) Y_{m}^{\prime}(x)-Y_{m}(x) J_{m}^{\prime}(x)=2 / \pi x$ then gives

$$
G=\frac{2}{C^{2} \pi \sqrt{\frac{\mu_{0}}{\epsilon_{0}}}\left(k_{0} l\right)^{2}} \sum_{m=-\infty}^{m=\infty} \frac{\left[\sin ^{2}\left(\frac{m \phi_{0}}{2}\right)\right]}{\left(\frac{m \phi_{0}}{2}\right)} \int_{-1}^{1} \frac{\cos ^{2}\left(\frac{k_{0} l}{2} y\right) d y}{\left[y^{2}-\pi^{2} /\left(k_{0} l\right)^{2}\right]^{2}\left|H_{m}(2) \prime\left[C \sqrt{1-y^{2}}\right]\right|^{2}}
$$

Expression (16) is valid for any size axial slot provided it is thin enough so that the tangential electric fields across it are indeed given by (1) and (2).

If one now considers the special case of a very thin $\left(\frac{\sin \left(\frac{m \phi_{0}}{2}\right)}{\left(\frac{m \phi_{0}}{2}\right)} \approx 1\right)$ resonant half-wavelength

$\left(k_{0} l=\pi\right)$ slot then $G$ becomes, after making the change of variables $y=\cos \theta$, 


$$
G \mid=\frac{4}{\pi^{3} C^{2} \sqrt{\frac{\mu_{0}}{\epsilon_{0}}}} \int_{0}^{\pi} \frac{\cos ^{2}\left(\frac{\pi}{2} \cos \theta\right)}{\sin ^{3} \theta} \sum_{m=0}^{\infty} \frac{1}{\left(1+\delta_{0}^{m}\right)\left|H m^{(2)^{\prime}}(C \sin \theta)\right|^{2}} d \theta
$$

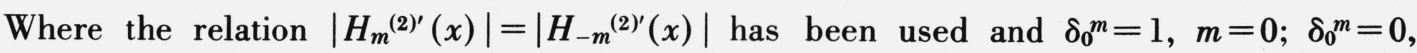
$m \neq 0$, is the Kronecker delta. Expression (17) is identical to that obtained by Wait [1955, eq (15)] using integration over physical space of the radiation fields. A plot of $G$ versus $C$ is given in Wait's work [1955, fig. 11; 1959, fig. 10] and shows that for $C \geqslant 10, G$ is within 10 percent of the conductance of the same slot cut in an infinite flat ground plane.

In conclusion, from the above, it is seen that use of Parseval's theorem offers another method for determining the radiation conductance of a slot on a perfectly conducting cylinder. It has the advantage of not requiring a determination of the spatial variations of the fields as is required when using integration of Poynting's vector, since it only involves operations with the cylindrical transforms of the fields. Additionally, it can also be used to determine the susceptance of the slot by extraction of the imaginary part of the bracket in (10). This method can also be extended to the case of a slot on a coated cylinder. This is presently being done.

\section{Appendix A. Parseval's Theorem for Power Flow Through an Axial Slot}

Consider an axial slot on a metal cylinder as depicted in figure 1 . If the tangential electric fields are given by (1) and (2), it follows that the power flowing through the slot is given by (8). Now since $E_{\phi}(a, \phi, z)$ is zero off the slot, (8) can also be written as an integration over the entire cylindrical surface $\rho=a$, i.e.,

$$
P=\operatorname{Re}\left\{\frac{a}{2} \int_{-\infty}^{\infty} \int_{-\pi}^{\pi} E_{\phi}^{*} H_{z} d \phi d z\right\}
$$

where in both (8) and (18) the arguments $(a, \phi, z)$ on the fields are understood. Now $H_{z}$ can be expressed as (6) and similarly

$$
E_{\phi}^{*}(a, \phi, z)=\sum_{m^{\prime}=-\infty}^{m^{\prime}=\infty} \int_{-\infty}^{\infty} \bar{E}_{\phi m^{\prime}}^{*}\left(h^{\prime}\right) e^{j h^{\prime} z} d h^{\prime} e^{j m^{\prime} \phi}
$$

where $h^{\prime}$ and $m^{\prime}$ are dummy variables.

Substituting (6) and (19) into (18) gives

$P=\operatorname{Re}\left\{\frac{a}{2} \int_{-\infty}^{\infty} \int_{-\pi}^{\pi}\left\{\sum_{m^{\prime}=-\infty}^{m^{\prime}=\infty} \int_{-\infty}^{\infty} \overline{E_{\phi m^{\prime}}^{*}}\left(h^{\prime}\right) e^{j h^{\prime} z} d h^{\prime} e^{j m^{\prime} \phi} \sum_{m=-\infty}^{m=\infty} \int_{-\infty}^{\infty} \bar{H}_{z m}(h) e^{-j h z} d h e^{-j m \phi} d \phi d z\right.\right.$.

Rearranging:

$P=\frac{a}{2} \operatorname{Re}\left[\sum_{m=-\infty}^{m=\infty} \int_{-\infty}^{\infty} \bar{H}_{z m}(h)\left\{\sum_{m^{\prime}=-\infty}^{m^{\prime}=\infty} \bar{E}_{\phi m^{\prime}}^{*}\left(h^{\prime}\right) \cdot\left[\int_{-\infty}^{\infty} \int_{-\pi}^{\pi} e^{j z\left(h^{\prime}-h\right)} e^{j \phi\left(m^{\prime}-m\right)} d \phi d z\right] d h^{\prime}\right\} d h\right]$ 
and recognizing that

$$
\begin{gathered}
\int_{-\pi}^{\pi} e^{j \phi\left(m^{\prime}-m\right)} d \phi=2 \pi \delta_{m}^{m^{\prime}}= \begin{cases}2 \pi & m^{\prime}=m \\
0 & m^{\prime} \neq m\end{cases} \\
\int_{-\infty}^{\infty} e^{j z\left(h^{\prime}-h\right)} d z=2 \pi \delta\left(h^{\prime}-h\right)
\end{gathered}
$$

where $\delta_{m}^{m^{\prime}}$ is the Kronecker delta and $\delta\left(h^{\prime}-h\right)$ is the Dirac delta function, which when substituted into (21) gives

$P=(2 \pi)^{2} \frac{a}{2} \operatorname{Re} \sum_{m=-\infty}^{m=\infty} \int_{-\infty}^{\infty} \bar{H}_{z_{m}}(h)\left\{\sum_{m^{\prime}=-\infty}^{\infty} \int_{h^{\prime}=-\infty}^{\infty} \bar{E}_{\phi_{m^{\prime}}}\left(h^{\prime}\right) \delta_{m}^{m^{\prime}} \delta\left(h^{\prime}-h\right) d h^{\prime}\right\} d h$.

Since the delta function has the property $\int_{\infty}^{\infty} f(x) \delta\left(x-x_{0}\right) d x=f\left(x_{0}\right)$, the above expression becomes

$$
P=\frac{a}{2}(2 \pi)^{2} \operatorname{Re} \sum_{m=-\infty}^{m=\infty} \int_{-\infty}^{\infty} \bar{E}_{\phi m}^{*}(h) \bar{H}_{z_{m}}(h) d h=\frac{a}{2}(2 \pi)^{2} \operatorname{Re} \sum_{m=-\infty}^{m=\infty} \int_{-\infty}^{\infty} \bar{E}_{\phi}^{*} \bar{H}_{z} d h .
$$

This result is a statement of Parseval's theorem in terms of the cylindrical transforms of $E_{\phi}$ and $H_{z}$.

Acknowledgment is given to H. Hodara of NESCO for discussions leading to the derivation of (25).

\section{References}

Harrington, R. F. (1961), Time harmonic electromagnetic waves, pp. 180-186 (McGraw Hill Book Co., Inc., New York, N.Y.). Wait J. R. (Dec.' 1955), Radiation characteristics of axial slots on a conducting cylinder, Wireless Engineer, 32, No. 12, $316-322$.

Wait, J. R. (1959), Electromagnetic radiation from cylindrical structures (Pergamon Press, New York, N.Y.).

(Paper 69D3-482) 